\title{
Aetiological analysis of primary amenorrhea: a retrospective study
}

\author{
Uma Jain $^{1 *}$, Preeti Gupta ${ }^{2}$
}

\begin{abstract}
${ }^{1}$ Department of Obstetrics and Gynaecology, GMC associated with DH Shivpuri, Madhya Pradesh, India ${ }^{2}$ Department of Obstetrics and Gynaecology, Gupta Maternity hospital and Infertility centre, Morar, Madhya Pradesh, India.
\end{abstract}

Received: 03 December 2020

Revised: 01 January 2021

Accepted: 04 January 2021

\author{
*Correspondence: \\ Dr. Uma Jain, \\ E-mail: ujain880@gmail.com
}

Copyright: (c) the author(s), publisher and licensee Medip Academy. This is an open-access article distributed under the terms of the Creative Commons Attribution Non-Commercial License, which permits unrestricted non-commercial use, distribution, and reproduction in any medium, provided the original work is properly cited.

\begin{abstract}
Background: Primary amenorrhea is defined as the failure to reach menarche. Primary amenorrhea is the lack of menses by age 15 with secondary sex characteristics, or at 13 with absence of secondary sex characteristics. Objective was to determine various etiological factors of primary amenorrhea in Gynecological practice.

Methods: A retrospective study was conducted in a Gynae clinic in Shivpuri and a Maternity hospital in Gwalior from January 2015 to December 2019.

Results: A total of 57 of the patients were evaluated in the study period. Most of the patients were unmarried adolescent girls $(71.92 \%)$. The most common presenting symptom was, not attained menarche $(36.84 \%)$. Based on the presence or absence of breast and uterus: group, I - breast present and uterus present 13 cases (22.77\%), group II breast present and uterus absent 26 cases $(45.61 \%)$, group III - breast absent and uterus present 17 cases $(29.79 \%)$, group IV - breast absent and uterus absent 1 case $(1.75 \%)$ were present. The most common etiological factors were Mullerian Agenesis 22 cases (38.60\%) and Gonadal dysgenesis 7 cases (12.28\%). (56.14 \%) cases were normogonadotropic, followed by $(15.78 \%)$ cases were of Hypergonadotropic hypogonadism, (14.03\%) hypogonotropic hypogonadism and (14.03\%) were of hyperendrogenism and others causes. In cases of Mullerian abnormalities, in findings of renal ultrasound or IVP $(72.72 \%)$ cases were normal.

Conclusions: The most common etiological factors of primary amenorrhea were Mullerian Agenesis. Amenorrhea is a common problem encountered by the primary care physician. A thorough history and clinical examination are needed for differential diagnosis.
\end{abstract}

Keywords: Primary amenorrhea, Hypergonotropic hypogonadism, Mullerian agenesis.

\section{INTRODUCTION}

Amenorrhea is the absence of spontaneous menses in a woman of reproductive age. It is divided into primary amenorrhea and secondary amenorrhea.

Primary amenorrhea is the failure to reach menarche. Primary amenorrhea is the lack of menses by age 15 with secondary sex characteristics, or at 13 with the absence of secondary sex characteristrics. ${ }^{1,2}$
Secondary amenorrhea is characterized as the cessation of previously regular menses for three months or previously irregular menses for six months. ${ }^{3}$

According to World health organization estimates, amenorrhea stands as the sixth largest major cause of female infertility and affects $2-5 \%$ of all women of childbearing age. ${ }^{4}$ 
A study in India indicated that $11.1 \%$ of adolescent girls, who presented with gynaecological complaints of menstrual disorders, had primary amenorrhea. However, the incidence of the disease in women, in general, is less than $1 \% .^{5}$

Puberty is the physical emotional and sexual transition from childhood to adulthood. It involves five changesbreast, pubic hair, axillary hair development, the growth spurt, and the onset of menstruation. Thelarche (Breast development) is the first sign of normal sexual development which is estrogen dependent, followed by the pubarche or adrenarche (pubic and axillary hairs) which is adrenal androgen and estrogen-dependent followed by menarche (menstruation).

There are five basic factors involved in the onset and continuation of menstruation. These are anatomical patency of genital tract, normal female chromosomal pattern (46XX), synchronized regulatory 'feed-back' mechanism of hypothalamic-pituitary-ovarian axis; active support by two other ancillary endocrine glands namely, thyroid and adrenal cortex and responsive endometrium. Defect in any one or more of these factors will be responsible for amenorrhea. 6

Genetic and receptor enzyme defects also play an essential role in the causation of primary amenorrhea.

Errors in the fetus, errors in the development of gonads, Mullerian and Wolffian systems, and the urogenital sinus are the most frequent causes of primary amenorrhea. Nearly all individuals without secondary sex characteristics have a genetic basis for primary amenorrhea.

Although a rare disorder, the most common cause of primary amenorrhea involves gonadal failure, congenital absence of the uterus and vagina, and constitutional delay.

There are three primary ways to classify primary amenorrhea.

(1st) The world health Organization (WHO) has summarized the causes 1) WHO group I there is no evidence of endogenous estrogen production, normal or low FSH levels, normal Prolactin levels, and no evidence of a lesion in the hypothalamic-pituitary region; 2) WHO group II is associated with evidence of estrogen production and normal levels of prolactin and FSH 3) WHO group III involves elevated serum FSH levels indicating gonadal failure. ${ }^{7}(2 \mathrm{nd})$ the practice committee of the American Society for Reproductive Medicine (2008) has summarized thecause: ${ }^{8}$ Anatomic defects of the genital tract, hypothalamic/pituitary causes, ovarian insufficiency, endocrinopathies, chronic oligo - or anovulation (3rd) clinically the causes ofprimary amenorrhea can be classified according to the presence or absence of secondary sexual characteristics (development of breast, pubic hair, and axillary hair) and presence or absence of uterus. Group 1. breast present, uterus present, group 2. breast present, uterus absent, group 3. breast absent and uterus present, group 4 breast absent, uterus absent.

In the workup of primary amenorrhea, history, physical examination, laboratory investigations, ultrasound examinations, MRI Karyotyping, and others are necessary. The workup of primary amenorrhea included the following.

History $^{\text {9, } 10}$

Family history of primary or secondary amenorrhea, pubertal history, delayed growth, and delayed menarche, genetic defect, endocrinopathies, mental retardation, consanguinity, and any other significant illness was also elicited.

Personal History: Patients were asked about their eating habits, history of Nutritional status, Bulimia, anorexia, weight changes, exercise pattern, psychosocial stress, Scholastic performance, history of major psychiatric disorders.

History of puberty milestones like thelarche, pubarche, menarche, history of cyclic abdominal pain, history of chronic illness like thyroid, renal, diabetes, sarcoid, history of headaches, vomiting, any disorder of smell perception, decreased peripheral vision, head trauma, visual disturbances, history of medications like hormones, antidepressants, anxiolytics, history of hot flushes, vaginal dryness, history of breast discharge or lactation, history of hirsutism or acne, feeling of excessively hot or cold, palpitations, diarrhoea, constipation, tremor depression, skin changes, history of neonatal / childhood encephalitis, history of Irradiation or chemotherapy, history of hospitalization and surgeries, history of sexual activities, Uterine instrumentation or any other operation, prior diagnostic studies and results were also recorded.

History was elaborated regarding withdrawal bleeding to progesterone or estrogen\&progesterone.

\section{Physical examination}

In depth attention should be paid to the signs of sex hormone activity (e.g., adequate development of the breasts, pubic and axillary hair shows normal ovarian function and points to an extragonadal cause for amenorrhea). Various causes of primary amenorrhea are categorized in four groups, according to clinical and ultrasound findings based on the presence or absence of breast and uterus. A simplified clinical evaluation of the aetiology of primary amenorrhea is presented in Table 1 . 
Table 1: A simplified clinical evaluation of the aetiology of primary amenorrhea. ${ }^{11-13}$

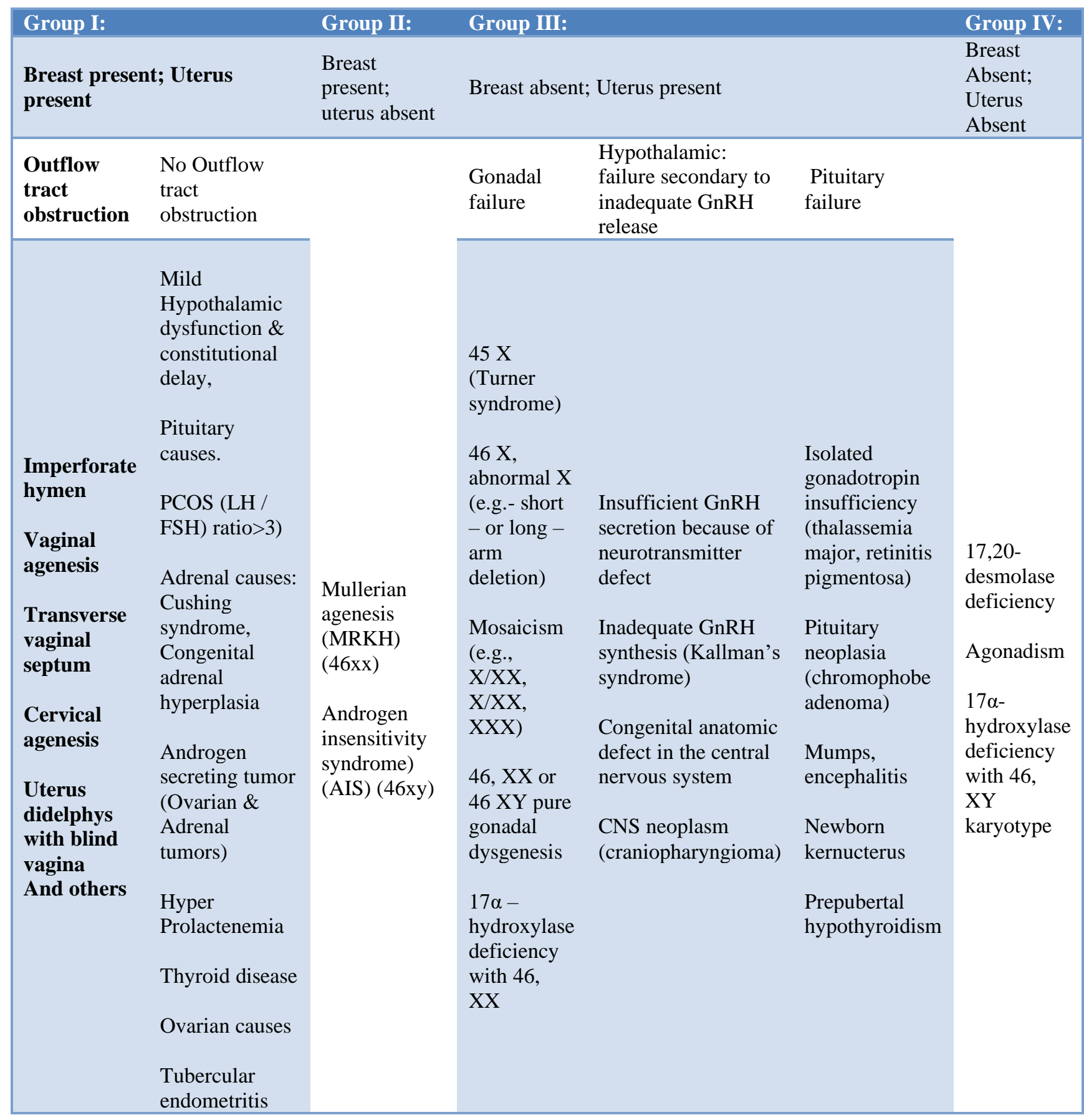

In general, these finding should be included in the observation.

Physical examination included an examination of height, weight, anthropomorphic measurement, growth chart, BMI (body mass index), body habitus, pulse BP; signs of an eating disorder, hypothermia, bradycardia, hypotension reduced subcutaneous fat; assessment of secondary sexual characters. Tanner staging of breast and pubic hairs and axillary hair; thyroid palpation; increase facial hair, change in voice, and other signs of virilization, hirsutism, deep voice, acne, masculine body, clitoris hypertrophy; Galactorrhea; presence of dysmorphic features such as a webbed neck, short stature, low hairline, broad shield-like chest with widely spaced nipples and other stigmata of Turner syndrome. Striae, buffalo hump, central obesity (signs of cushing syndrome), scoliosis, and stigmata of other diseases are also noted; neurological examination: visual field, sense of smell, optic fundus, and others; abdominal examination: presence of a mass, arising out of the pelvis, (hematometra/ hematocolpos, pregnancy, ovarian tumour). Palpation of inguinal areas- for hernia or swelling due to the presence of gonads (testis). Pelvic 
examination: local and per rectal examination: the ambiguity of external genitalia or infantile external genitalia,

Clitromegaly, bulging bluish membrane, labioscrotal fusion to no patency of the vagina, reddened or thin vaginal mucosa, depth of vagina, short vaginal length, transverse vaginal septum, presence or absence of cervix, presence or absence of uterus (normal/ infantile /rudimentary), presence of a mass in the fornix. Any ovarian tumours and to rule out pregnancy.

\section{Investigation}

\section{Laboratory investigations}

The initial workup included follicle-stimulating hormone, serum luteinizing hormone, and estradiol to determine whether the patient was, normogonotropic (FSH, LH), hypogonadotropic (FSH, LH), or Hypergonadotropic hypogonadism (FSH, LH). Prolactin and thyroidstimulating hormone levels. Estradiol, serum-free and total testosterone, and dehydroepiandrosterone sulfate (DHEAS). The 17-OHP level was also studied when indicated.

A complete blood count, Mantoux test, an X-ray of the chest is to be done if history or examination was suggestive of chronic disease.

Urine pregnancy test or beta HCG, Progesterone challenge test, estrogen, and progesterone withdrawal test are also an essential part of the investigation protocol.

\section{Pelvic ultrasonography (transabdominal or transvaginal)}

It confirms the presence of a uterus, ovary, and any reproductive tract anomaly.

\section{MRI}

In cases where USG was inconclusive, MRI was done to visualize Mullerian structure, gonads, and renal anomalies, laparoscopy and hysteroscopy were also done if it is indicated.

\section{Cytogenetic study (karyotyping)}

It was done in cases of primary gonadal failure and those with symptoms of androgen excess.

Clinically the diagnosis of primary amenorrhea is not always possible; the treating doctor must be concerned with various investigations and disorders involving many organ systems. A proper approach to diagnosis is a must.

Early diagnosis and timely intervention are necessary to prevent long-term health and social consequences. ${ }^{14}$
As soon as the aetiology is established in a particular case, the patient can be counselled regarding the prognosis and future fertility options..$^{15}$

\section{METHODS}

A retrospective study was conducted in a Gynae clinic in Shivpuri and a Maternity hospital \& Infertility centre in Gwalior. All primary amenorrhea cases who attended the Gynaecologic OPD from January 2014 to December 2019 were included in the study. Fifty-seven patients with primary amenorrhea were retrospectively categorized on clinical and ultrasound findings in four groups based on the presence or absence of breast and uterus.

Group 1. breast present, uterus present, Group 2 breast present, uterus absent, Group 3 breast absent, uterus present, Group4 breast absent, uterus absent

Patients were also categorized according to their gonadotropin level 1) normogonadotropic 2)

Hypergonadotropic hypogonadism 3) hypogonadotropic hypogonadism 4) hyper androgenic and others.

Cases were analyzed according to history, clinical examination, physical examination, development of secondary sexual characteristics, pelvic and rectal Examination, biochemical and hormonal investigations, progesterone challenge test, progesterone and estrogens challenge test, pelvic ultrasound, Karyotyping, MRI, and other investigations if required proper approach to diagnosis is made according to Table 2.

The data was collected from the medical records of the 57 patients. All the data were analyzed using IBM SPSS ver. 20 software. Cross tabulation and frequency distribution were used to prepare the table. Data are expressed as numbers and percentages.

\section{RESULTS}

Total of 57 of the patients were evaluated in the study period.

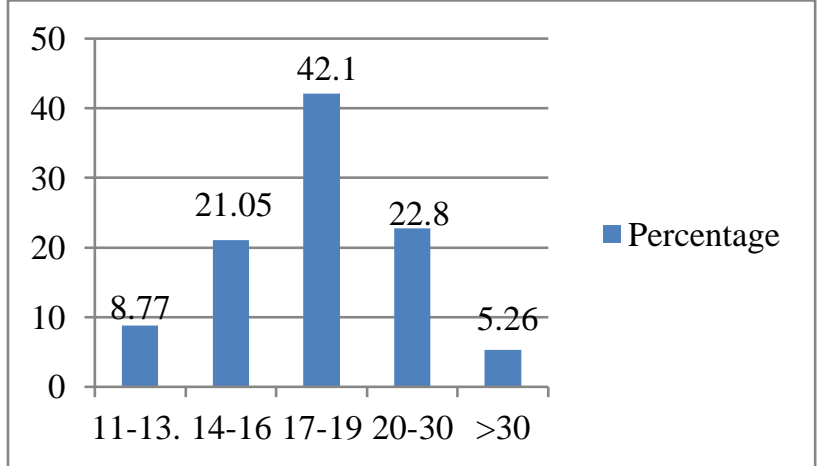

Figure 1: The most common reporting age was 17-19 years $(42.1 \%)$. 


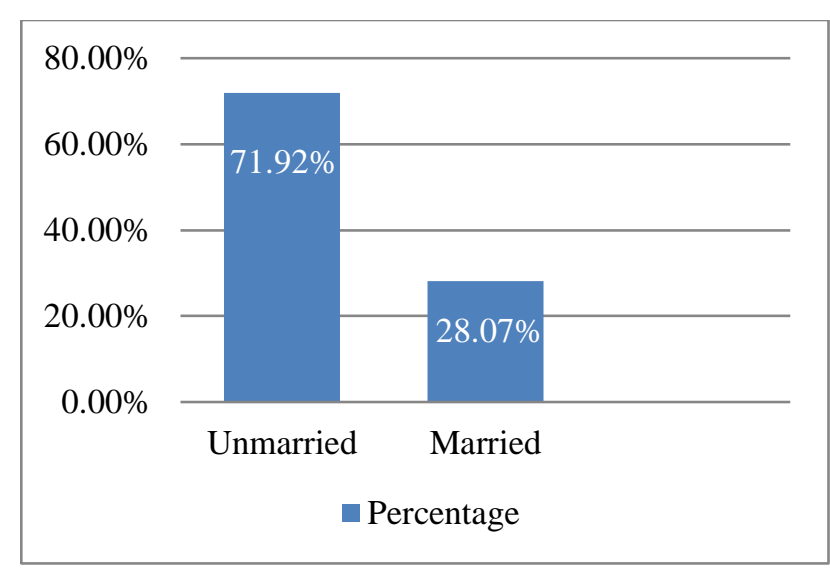

Figure 2: Most of the patients were unmarried adolescent girls $\mathbf{7 1 . 9 2 \%}$.
Table 2 showing the most common causes of primary amenorrhea according to clinical features of the patient.

In this study in group I - breast present and uterus present 17 cases $(22.77 \%)$, group II- breast present and uterus absent 26 cases (45.61) group III - breast absent and uterus present 17 cases (29.79) group IV - breast absent and uterus absent 1 case (1.75) was present. The most common causative factor was (MRKH syndrome) 22 cases $(38.60 \%)$, followed by Gonadal dysgenesis 7 cases (12.28\%), followed by Complete Androgen insensitivity syndrome 4 cases $(7.02 \%)$, followed by others 25 cases $(43.87 \%)$.

In our study, the most common presenting symptoms were not attained menarche $(36.84 \%)$ followed by the false history of menarche and stoppage menstruation $(19.29 \%)$.

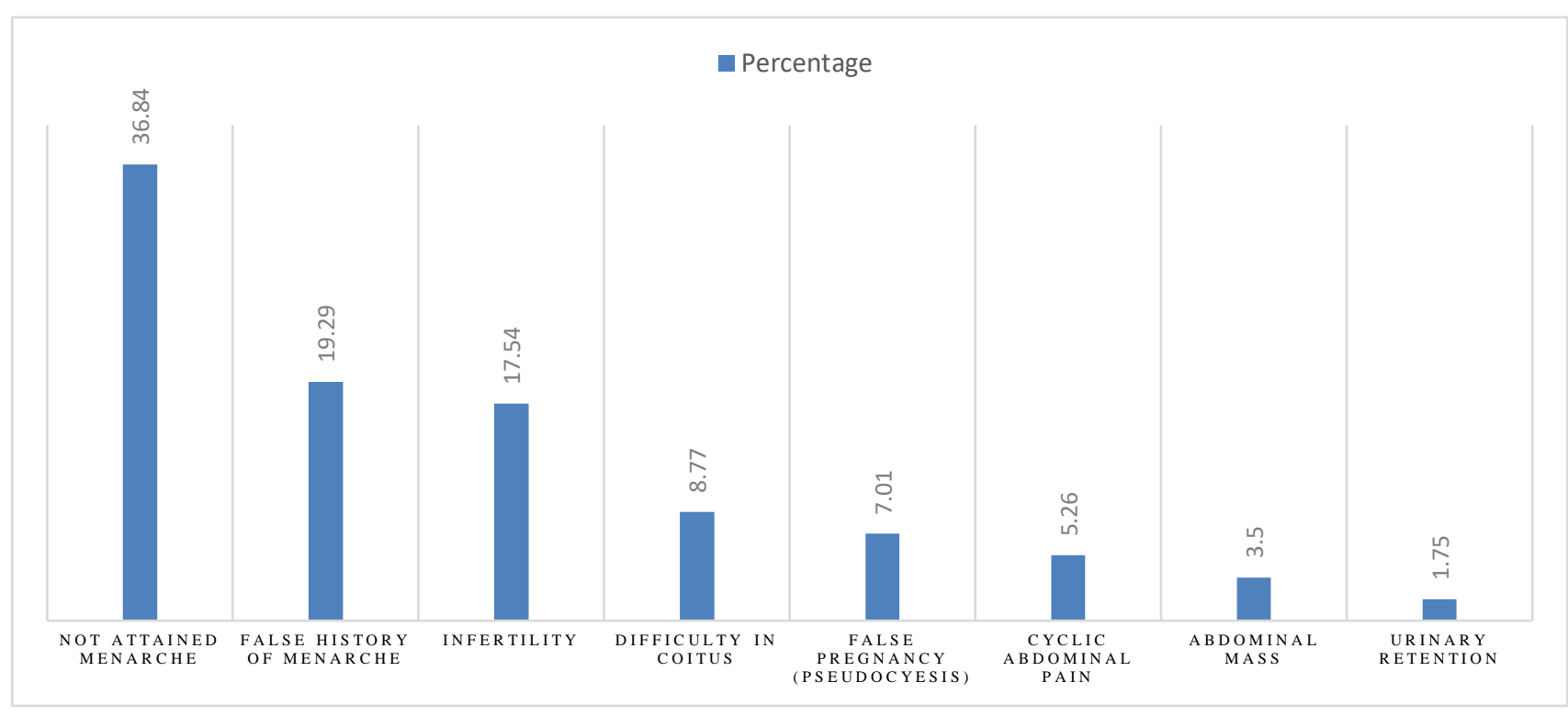

Figure 3: Most common presenting symptom.

Table 2: Causes of primary amenorrhea.

\begin{tabular}{|c|c|c|}
\hline Causes of Primary Amenorrhea can be subdivided into & No. & $\%$ \\
\hline Group I - breast present and uterus present & 13 & 22.77 \\
\hline \multicolumn{3}{|l|}{ End organ failure / outflow tract obstruction } \\
\hline Imperforate hymen & 2 & 3.51 \\
\hline Cervicovaginal Agenesis & 1 & 1.75 \\
\hline Transverse vaginal septum & 1 & 1.75 \\
\hline Uterus didelphys with blind vagina & 1 & 1.75 \\
\hline Tubercular endometritis & 1 & 1.75 \\
\hline \multicolumn{3}{|c|}{ Hyper androgenism \& others (hyperprolactinimia \& hypothyroidism) } \\
\hline PCOS & 2 & 3.51 \\
\hline Congenital adrenal hyperplasia & 0 & 0 \\
\hline Androgen secreting tumour & 1 & 1.75 \\
\hline \multicolumn{3}{|l|}{ Others } \\
\hline Primary Hypothyroidism & 1 & 1.75 \\
\hline Hyperprolactinemia & 2 & 3.51 \\
\hline Prolactinoma & 1 & 1.75 \\
\hline
\end{tabular}

Continued. 


\begin{tabular}{|c|c|c|}
\hline Causes of Primary Amenorrhea can be subdivided into & No. & $\%$ \\
\hline Group II - breast present and uterus absent & 26 & 45.61 \\
\hline Mullerian Agenesis (MRKH syndrome) & 22 & 38.60 \\
\hline Complete Androgen insensitivity syndrome & 4 & 7.02 \\
\hline Group III - breast absent and uterus present & 17 & 29.79 \\
\hline \multicolumn{3}{|l|}{ Ovarian causes } \\
\hline Gonadal dysgenesis & 7 & 12.28 \\
\hline Pure Gonadal dysgenesis (46XX) & 4 & 7.02 \\
\hline 46xyGonadal dysgenesis, Swyerrs syndrome (46xy) & 1 & 1.75 \\
\hline Turner (45X0 / Mosaic) & 1 & 1.75 \\
\hline Triple X syndrome & 1 & 1.75 \\
\hline Premature ovarian failure & 2 & 3.51 \\
\hline - Post Chemotherapy & 1 & 1.75 \\
\hline \multicolumn{3}{|l|}{ - Post irradiation } \\
\hline - Idiopathic & 1 & 1.75 \\
\hline Hypothalamic and Pituitary causes & 8 & 14.03 \\
\hline Constitutional delay in growth and development & 3 & 5.26 \\
\hline Congenital gonadotropin deficiency (Kallman syndrome) & 0 & 0 \\
\hline Idiopathic gonadotropin deficiency & 1 & 1.75 \\
\hline Irradiation & 1 & 1.75 \\
\hline Chronic debilitating diseases & 2 & 3.51 \\
\hline Hypothalamic dysfunction (severe cases) & 1 & 1.75 \\
\hline Group IV- breast absent and uterus absent & 1 & 1.75 \\
\hline
\end{tabular}

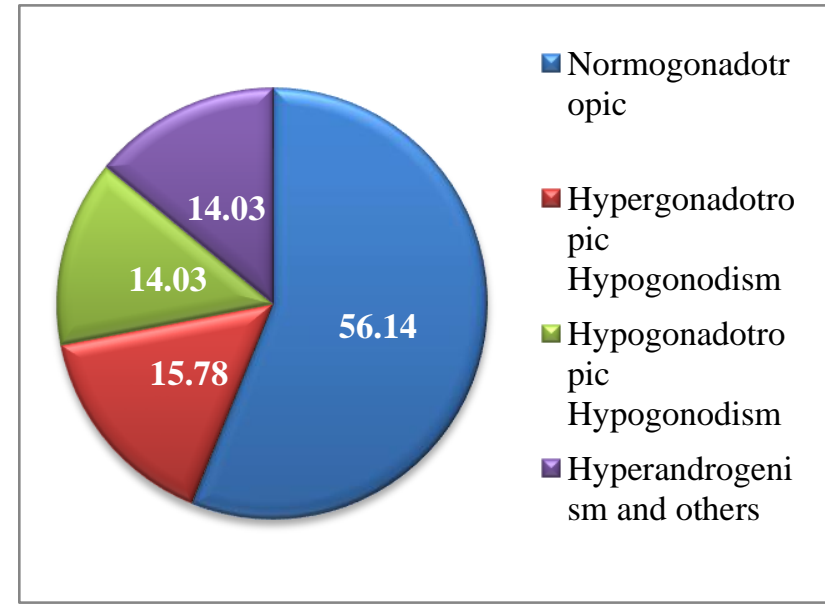

Figure 4: Causes of primary amenorrhea according to hormone level.

In this study according to the hormonal milieu the most common cause of primary amenorrhea was Normo gonadotropic $56.14 \%$, followed by hyper gonadotropicin $15.78 \%$ cases, hypo gonadotropic $14.03 \%$, and hyper androgenism and others $14.03 \%$ (Figure 4).

In findings of renal ultrasound or IVP with Mullerian abnormality (in 22 Cases) $72.72 \%$ were normal, $9.09 \%$ were having absent one kidney, and $9.09 \%$ were one ectopic kidney and other normal, bilateral ectopic kidney was found in $4.54 \%$ cases (Figure 5).

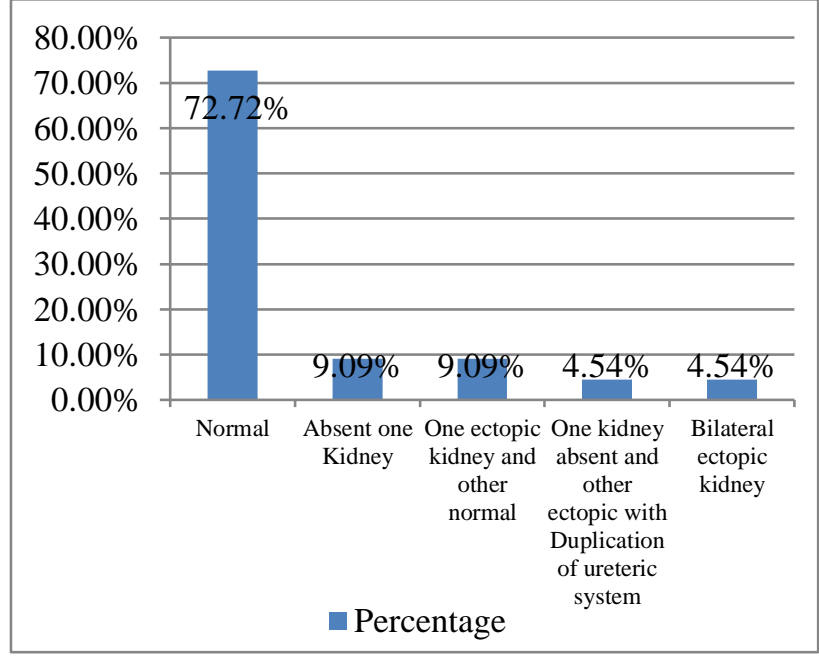

Figure 5: In findings of renal ultrasound or IVP with Mullerian abnormality (in 22 cases) $72.72 \%$ were normal, 9.09\% were having absent one kidney, and 9.09\% were one ectopic kidney and other normal, Bilateral ectopic kidney was found in $4.54 \%$ cases.

\section{DISCUSSION}

Most primary amenorrhea cases can be diagnosed clinically from the detailed history and physical examination and general investigative approach detailed above. Rigid adherence to the protocol for the investigation of amenorrhea will usually result in an efficient and rapid diagnosis, minimize expenses, and identify those requiring referral to specialized centres. 
Previous studies have been reported from all parts of the world, indicating the frequency of various etiologies and cytogenetic abnormalities in primary amenorrhea cases. Gonadal dysfunction and outflow tract anomalies have been considered as the commonest factor for primary amenorrhea worldwide.

A total of 57 of the patients was evaluated in the study period. The most common age of reporting was 17-19 years $(42.1 \%)$ which was a correlation with a study reported by Bhuyan et al in which the mean age of presentation for primary amenorrhea was 17.23 \pm 4.2 years. $^{16}$

In our study, the most common presenting symptoms were not attained menarche $(36.84 \%)$ followed by the false history of menarche and stoppage of menstruation (19.29\%) which was comparable with, (46.66\%) found with a complaint of not attaining the menarche in the study of Asma Akhtar and Sudhir. ${ }^{17}$

In this study in group I - breast present and uterus present 13 cases $(22.77 \%)$, group II - breast present and uterus absent 26 cases (45.61) group III - breast absent and uterus present 17 cases (29.79) group IV - breast absent and uterus absent 1 case (1.75) was present. This study was not consistent with another study in which $(35.48 \%)$ patients were in group I, $(14.51 \%)$ patients were in group II, (46.77\%) patients were in group III, and (3.22\%) patients were in group IV. ${ }^{18}$

In this study, the most common causative factor was Mullerian agenesis (MRKH syndrome) in 22 cases $(38.60 \%)$ which are in correlation with the study from Thailand Prasong et al (39.7\%), from AIIMS New Delhi, (Alka Kriplani), (47\%), the study of GS Anitha et al. $(32.50 \%)$, and the study of Quorrata et al (36.3\%). ${ }^{19-22}$ The incidence of Mullerian agenesis was lesser than the study of (66\%) Asma Akhtar, Sudhir Balampa, Rabindran, Pavani (65.78\%). ${ }^{17,23}$

The other most common causative factor was Gonadal dysgenesis 7 cases (12.28\%). Our study was comparable with Asma Aktar, Sunita Sudhir. Where gonadal dysgenesis was found in (13.33) \% cases. ${ }^{17}$ Our study was not comparable with the study of Prasong et al where gonadal dysgenesis was $(35.3 \%){ }^{19}$

The other most common causative factor was Complete Androgen insensitivity syndrome $7.02 \%$ which was comparable with the study of Asma Akhtar, Sunita Sudhir Pwhere the Androgen insensitivity was $(6.66 \%) .17$

In this study according to the hormonal milieu, the most common cause of primary amenorrhea was normo gonadotropic $56.14 \%$, followed by hyper gonadotropic in $15.78 \%$ cases, was comparable with the study of Kumar et al. in which normogonadotropic was the commonest and hypergonadotropic hypogonadism was found in
(16.60\%) cases. $^{24}$ Our study was not comparable with another study from Kashmirin which $40.50 \%$ of patients had hypergonadotropic hypogonadism, (34.17\%) had hypogonadotropic hypogonadism, and in $12.65 \%$ Mullerian agenesis was found. ${ }^{25}$ Our study was also not consistent with the study of Rattanachaiyanont et al and GS Anitha et al in which they found hypergonadotropic, hypogonadism in $32.69 \%$ and $32.50 \%$ of cases respectively. ${ }^{26,20}$

In findings of renal ultrasound or IVP with Mullerian abnormality (in 22 cases) $72.72 \%$ were normal, 9.09\% were having absent one kidney, and $9.09 \%$ were one ectopic kidney and other normal. This was comparable with another study in which $72 \%$ of cases were normal, $12 \%$ of cases had absent one kidney, and (9\%) cases had one ectopic and another normal kidney. ${ }^{16}$

The treatment for amenorrhea depends on the underlying cause and the health status and goals of the individual.

In our study, some cases were referred to the higher centre to an endocrinologist, psychologist, and senior gynaecologist for further medical and surgical treatment.

\section{CONCLUSION}

In our study, Mullerian anomaly was the most common causative factor leading to amenorrhea followed by premature ovarian failure. Feeling of de feminization due to an absence of menstruation and/or undeveloped secondary sexual characteristics in primary amenorrheic patients makes them believe that they were "not normal women". Careful counselling has to be introduced not only to inform them of the nature of their disease but also to confirm their femininity.

A multidisciplinary team approach, including gynaecologist, endocrinologist, dietician, psychiatrist, and medical doctor, is needed to provide treatment and psychological support.

The goal of management should be to prevent problems related to the hypoestrogenic stages like osteoporosis and other related symptoms; to initiate gradual pubertal development to maintain a healthy hormonal milieu; to deal with sexual problems; to resume ovulation and menses; to deal with fertility problems; to manage problems from the nature of aetiology with or without the associated disease, (medical and surgical treatment); frequent re-evaluations and reassurance of the patients.

\section{ACKNOWLEDGMENTS}

Authors would like to thank Dr. Dilip Jain, Consultant Arihant Pathology, Shivpuri for helping me in this study.

Funding: No funding sources Conflict of interest: None declared

Ethical approval: Not required 


\section{REFERENCES}

1. Herman-Giddens ME, Slora EJ,Wasserman RC, Bourdony CJ, Bhapkar MV Koch GG, et al, (1997), Secondary sexual characteristics and menses in young girls seen in office practice: A study from the paediatric research in office setting network, paediatric, 1997;99:505-12.

2. Practice committee of the American Society for reproductive Medicine (2008) current evaluation of amenorrhea. Fertil Steril. 2008;90:219-25,

3. Doody KM. Carr Amenorrhea; obstetgynecol Clin, North Am, 1990;17:361-87.

4. Dutta UR, Ponnala R, Pidugu VK, Dalal AB. Chromosomal abnormalities in amenorrhea: a retrospective study and review of 637 patients in south India. Arch Iran Med. 2013;16(5):267-70.

5. Reeja T, Sunil S. Primary Amenorrhoea. Available at: http://www.medindia.net,/ Accessed on 03 April 2008.

6. Chakravarty BN;Amenorrhoea and Anovulation In: HansotiaMehroo, Desai Sadhana, Parihar Mandakini; Advanced Infertility Management; 1st edition; JAYPEE publication. 2002;11:99-117.

7. Insler V. Gonadotropin therapy: new trends and insights. Int J Fertil. 1988;33:85-97.

8. American College of Obstetricians and Gynaecologists. Amenorrhea (ACOG Technical Bulletin 128). Washington, D.C. ACOG, 1989.

9. David A. Klein, Poth. Merrily A.; Amenorrhea: An approach to Diagnosis and management; Am Fam Physician. 2013;87(11):781-8.

10. Condessa M. Curley; Amenorrhea; In: Carol Havens, Nancy D. Sullivan; Manual of Outpatient Gynecology, fourth edition; chapter. 11;105-18.

11. Lobo RA, Gershenson DM, Lentz GM, Valea F; Comprehensive Gynecology; 7th edition, 2017: Chapter 38; Primary and Secondary Amenorrhea and precocious puberty; 2017;7:829-52.

12. Kumar S, Padubidri VG, DaftrarSN y; editors.Shaw's textbook of Gynaecology; 17th edition; Primary and Secondary Amenorrhea. 17:141-54.

13. RatnamSS, Rao K, Bhasker, Arunkumaran S.; Obstetric, and gynecology for postgraduate; chapter 19; Amenorrhoea,1st edition 1993;1:201-21.

14. ErdalEren, HalilSaglam, Esra Deniz PapatyaCakir, Omer Tarim; Etiological evaluation of adolescents with primary amenorrhea; Ind J Padiatr 2014;81:8615 ,

15. Geckinli BB, Toksoy G, Sayar C, Soylemez MA, Yesil G, Aydin H et al. Prevalence of aneuploidies,
X-structural abnormalities and 46, XY sex reversal in Turkish women with primary amenorrhea of premature ovarian insufficiency. Eur $\mathrm{J}$ Obstet Gynecol Reprod Biol. 2014;182:211-5

16. Bhuyan AK, Sarma D, Saikia UK; Contemporary issues in Primary amenorrhea: an experience from a tertiary care center,Indian Journal of endocrinology and metabolism, 2012;16(8):387-8.

17. Akhtar A, Sudhir PS; Etiological Evaluation of Primary Amenorrhea in A Rural Teaching Hospital. J Biomedic Clinic Res. 2018;5(1):3-20.

18. Mashchak CA, Kletzky OA, Davajan V, Mishell DR $\mathrm{Jr}$; Clinical and laboratory evaluation of patients with primary amenorrhea; Obstet Gynecol. 1981;57(6):715-21

19. Tanmahasaut P, Rattanachaiyanont M, Dangrat C, Indhavivadhana S, AngsuwattanaS, Techatrisak K. Causes of primary amenorrhea: A report of 295 cases in Thailand; J Obstet Gynaecol Res. 2011;38(1):297301

20. Kriplani A, Goyal M, Kachhawa G, Mahey R, Kulshrestha V. Aetiology and management of primary amenorrhoea: A study of 102 cases at tertiary center. Taiwan Journal of Obstetrics \& Gynaecology. 2017;56(6):761-4.

21. Anitha GS, Tejeswini KK, MurtyGS; A clinical study of Primary Amenorrhea, J JSAFOG. 2015:7 (3): 158 .

22. Forhad QE, Tasnim S, Begum A. Primary Amenorrhoea-Analysis of 44 cases. Bangladesh J Obstet Gynaecol. 2008;23(2):46-50.

23. Balampa P, Rabindran, Pavani; Primary amenorrhoea - a single-center experience of 38 cases. 2015;1(1).

24. Kumar A, Mittal S. Primary amenorrhoea (analysis of 48 cases) J Indian Med Assoc. 1998;96(4):119-20.

25. Hassan Z, Abbas R, Abbas F, Gayas M; Etiological Profile of Primary amenorrhea in Kashmir Valley; Glob J Res Ana. 2019;8(6).

26. Rattanachaiyanont M, Kunathikom S, Angsuwattana $\mathrm{S}$, Mekmahan O, Techatraisak K, Mekmahan O, Karavagul C, Koanantakul S, et al. Primary amenorrhoea: a retrospective study at Siriraj Hospital. J Med Assoc Thai. 1997;80(10):619-25.

Cite this article as: Jain U, Gupta P. Aetiological analysis of primary amenorrhea: a retrospective study. Int J Reprod Contracept Obstet Gynecol 2021;10:484-91. 